\title{
Very sharp diffraction peak in nonglass-forming liquid with the formation of distorted tetraclusters
}

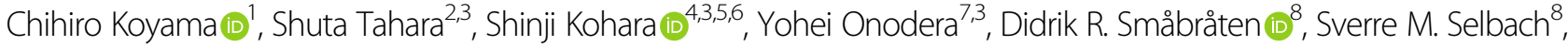 \\ Jaakko Akola9,10, Takehiko Ishikawa11,12, Atsunobu Masuno (13,3, Akitoshi Mizuno ${ }^{14}$, Junpei T. Okada ${ }^{15}$, \\ Yuki Watanabe ${ }^{16}$, Yui Nakata ${ }^{16}$, Koji Ohara (10) ${ }^{6}$, Haruka Tamaru', Hirohisa Oda', Ippei Obayashi 17,18,19, \\ Yasuyuki Hiraoka ${ }^{19,17,20,3}$ and Osami Sakata (1) ${ }^{4}$
}

\begin{abstract}
Understanding the liquid structure provides information that is crucial to uncovering the nature of the glass-liquid transition. We apply an aerodynamic levitation technique and high-energy $\mathrm{X}$-rays to liquid $(\Lambda)-\mathrm{Er}_{2} \mathrm{O}_{3}$ to discover its structure. The sample densities are measured by electrostatic levitation at the International Space Station. Liquid $\mathrm{Er}_{2} \mathrm{O}_{3}$ displays a very sharp diffraction peak (principal peak). Applying a combined reverse Monte Carlo - molecular dynamics approach, the simulations produce an $\mathrm{Er}-\mathrm{O}$ coordination number of 6.1, which is comparable to that of another nonglass-forming liquid, $I-\mathrm{ZrO}_{2}$. The atomic structure of $\mathrm{I}-\mathrm{Er}_{2} \mathrm{O}_{3}$ comprises distorted $\mathrm{OEr}_{4}$ tetraclusters in nearly linear arrangements, as manifested by a prominent peak observed at $\sim 180^{\circ}$ in the $\mathrm{Er}-\mathrm{O}-\mathrm{Er}$ bond angle distribution. This structural feature gives rise to long periodicity corresponding to the sharp principal peak in the X-ray diffraction data. A persistent homology analysis suggests that $I-\mathrm{Er}_{2} \mathrm{O}_{3}$ is homologically similar to the crystalline phase. Moreover, electronic structure calculations show that $I-\mathrm{Er}_{2} \mathrm{O}_{3}$ has a modest band gap of $0.6 \mathrm{eV}$ that is significantly reduced from the crystalline phase due to the tetracluster distortions. The estimated viscosity is very low above the melting point for $1-\mathrm{ZrO}_{2}$, and the material can be described as an extremely fragile liquid.
\end{abstract}

\section{Introduction}

Determining the liquid structure is the first step in understanding the nature of glass-liquid transitions. However, a diffraction measurement of liquid provides very limited structural information because the liquid structure lacks long-range periodicity, and a Fourier transform of the diffraction data provides only pairwise correlations. Moreover, high-quality measurements are difficult to obtain at high temperatures.

Since glasses play an important role in technology, glass formation has been studied extensively. Zachariasen ${ }^{1}$ and

\footnotetext{
Correspondence: Chihiro Koyama (koyama.chihiro@jaxa.jp) or

Shinji Kohara (KOHARA.Shinji@nims.go.jp)

${ }^{1}$ Human Spaceflight Technology Directorate, Japan Aerospace Exploration

Agency (JAXA), Tsukuba 305-8505, Japan

${ }^{2}$ Department of Physics and Earth Sciences, Faculty of Science, University of

the Ryukyus, Nakagami-gun, Okinawa 903-0213, Japan

Full list of author information is available at the end of the article

These authors contributed equally: Chihiro Koyama, Shuta Tahara
}

$\mathrm{Sun}^{2}$ proposed the basic concepts of glass formation by classifying constituents into glass formers, glass modifiers, and intermediates. Furthermore, Angell ${ }^{3}$ introduced the concept of "fragility" in glass-forming liquids (GFLs). He interpreted the strong and fragile behavior of liquids in terms of topological differences in potential energy hypersurfaces of the configuration space. Typical strong liquids are $\mathrm{SiO}_{2}, \mathrm{GeO}_{2}$, and $\mathrm{B}_{2} \mathrm{O}_{3}$. Their networks are covalently bonded, and the viscosities show an Arrhenius temperature dependence. In contrast, typical fragile liquids are chalcogenides and iron phosphates, the networks of which are mostly ionic and the viscosities of which deviate significantly from the Arrhenius behavior. Many experimental and theoretical structural studies of liquids and glasses have been performed, and with the advent of advanced synchrotron and neutron sources and the development of high-performance computers, they have led to great progress in our understanding of liquid and 
glass structures ${ }^{4,5}$. The structural analysis of liquids with high melting points has advanced significantly with the advent of the levitation technique ${ }^{6}$, especially in combination with diffraction techniques ${ }^{6}$. The structure of a typical non-GFL, liquid ( $l-) \mathrm{Al}_{2} \mathrm{O}_{3}$, and its undercooled liquid have been studied extensively by X-ray diffraction ${ }^{7-10}$, neutron diffraction $^{9-11}$, and molecular dynamics (MD) simulations ${ }^{9-13}$. In addition to the $l-\mathrm{Al}_{2} \mathrm{O}_{3}$ structure, several structures of molten pure oxides with high melting points $\left(T_{\mathrm{m}}\right)$ have been studied recently. For example, structures of $\mathrm{UO}_{2}{ }^{14}$ and compounds in the $\mathrm{UO}_{2}-\mathrm{ZrO}_{2}$ system ${ }^{15}$ have been investigated for nuclear reactor accidents. The structures of $\mathrm{ZrO}_{2}{ }^{16-18}, \mathrm{HfO}_{2}{ }^{16}$, and lanthanide oxides ${ }^{17}$ have also been investigated to understand the fundamental properties of high-temperature liquids. Although these investigations are very important not only for materials science but also for preventing severe accidents, the research methods and data are still limited by the high melting points of the materials in question.

$\mathrm{Er}_{2} \mathrm{O}_{3}$ is a representative nonglass former that is commonly used as a refractory material and dopant for luminescent materials. Because $\mathrm{Er}_{2} \mathrm{O}_{3}$ has an extremely high melting point $\left(T_{\mathrm{m}}=2686 \mathrm{~K}\right)$, the difficulties in handling the liquid lead to problems in selecting suitable container materials that do not contaminate the sample. To avoid contact with other materials, levitation furnaces have been developed that enable us to measure precise synchrotron X-ray diffraction and thermophysical properties for liquids at extremely high temperatures ${ }^{6}$.

This article presents the results of accurate high-energy $\mathrm{X}$-ray diffraction and density measurements on containerless levitated $l$ - $\mathrm{Er}_{2} \mathrm{O}_{3}$ using an electrostatic levitation furnace (ELF) at the International Space Station (ISS) ${ }^{19}$, as it is impossible to measure density data on the ground. We also perform reverse Monte Carlo - molecular dynamics simulations and obtain persistence diagrams from topological analyses to demonstrate liquid properties at the atomic level, comparing $l-\mathrm{Er}_{2} \mathrm{O}_{3}$ with other non-GFLs and a typical GFL, $l-\mathrm{SiO}_{2}$. Furthermore, a sample of $l-\mathrm{Er}_{2} \mathrm{O}_{3}$ is simulated for a short period with the density functional molecular dynamics method to investigate the electronic structure and to obtain a realistic estimate of the viscosity above the melting point. The combination of an experiment and a simulation allows trends in single-component nonglass-forming liquid oxides to be identified, with a focus on atomic ordering and topology. Furthermore, the article compares the features of single-component nonglass-forming oxide liquids with those of other systems.

\section{Materials and methods Density measurement}

The density of liquid $(l-) \mathrm{Er}_{2} \mathrm{O}_{3}$ was measured with an ELF at the ISS. A sample of $2 \mathrm{~mm}$ in diameter was prepared by melting $\mathrm{Er}_{2} \mathrm{O}_{3}$ powder with a purity of $99.99 \%$ and solidifying it in an aerodynamic levitator. It was charged by friction or contact with other materials in the ISS-ELF and then levitated to the center between six electrodes that applied a Coulomb force. The sample position was stabilized by tuning the voltages between electrodes at $1000 \mathrm{~Hz}$ and monitoring the image of the sample backlit by a $\mathrm{He}-\mathrm{Ne}$ laser. The levitated sample was heated and melted by four $40 \mathrm{~W}$ semiconductor lasers $(980 \mathrm{~nm})$ under 2 atm of dry air. The temperature of the sample was measured by a pyrometer $(1.45-1.8 \mu \mathrm{m})$. It was calibrated using an emissivity calculated from the plateau temperature at recalescence and the reference value of the melting point (2686 K). After melting, the nonspherical sample became spherical upon cooling after shutting off the lasers. During cooling, the sample image was observed by an ultraviolet back light and a CCD camera. The pixel size was calibrated against an image of $2.0 \mathrm{~mm}$ stainless steel spheres, which were recorded under the same conditions as the sample. The sample volume was calculated from its diameter, obtained from the image. Then, the density was calculated from the volume and weight.

\section{High-energy synchrotron X-ray diffraction measurement}

The high-energy X-ray diffraction measurement of $l$ $\mathrm{Er}_{2} \mathrm{O}_{3}$ was performed at the BL04B2 beamline ${ }^{20}$ of SPring-8 using an aerodynamic levitator ${ }^{21}$. The energy of the incident $\mathrm{X}$-rays was $113 \mathrm{keV}$. The $2-\mathrm{mm} \mathrm{Er}_{2} \mathrm{O}_{3}$ sample was levitated in dry air and heated by a $200 \mathrm{~W} \mathrm{CO}$ laser. The temperature of the sample was monitored by a two-color pyrometer. The background of the instrument was successfully reduced by shielding the detector and by optimizing a beam stop. The measured X-ray diffraction data were corrected for polarization, absorption, and background, and the contribution of Compton scattering was subtracted using standard analysis procedures ${ }^{22}$. The corrected data sets were normalized to give the Faber-Ziman ${ }^{23}$ total structure factor $S(Q)$, and the total correlation function $T(r)$ was obtained by a Fourier transform of $S(Q)$.

\section{Molecular dynamics - reverse Monte Carlo simulation}

To determine the atomic configuration of $l-\mathrm{Er}_{2} \mathrm{O}_{3}$, a molecular dynamics - reverse Monte Carlo (MD-RMC) simulation was performed with 5000 particles in a cube to reproduce the X-ray $S(Q)$. The MD simulation was carried out with a Born-Mayer type of pairwise potential with a Coulomb interaction and a repulsive component, given by the following equation:

$$
U_{i j}(r)=\frac{e^{2}}{4 \pi \varepsilon_{0}} \frac{Z_{i} Z_{j}}{r}+B_{i j} \exp \left(-\frac{r}{\rho_{i j}}\right),
$$

where $r$ is the interatomic distance, $Z$ is the effective charge, $B$ is the repulsion, $e$ is the elementary charge $\left(Z_{\mathrm{Er}}=2.1, Z_{\mathrm{O}}=-1.4\right), \varepsilon_{0}$ is the permittivity of the 
Table 1 The parameters for the Born-Mayer potential used in the MD simulation.

\begin{tabular}{lll}
\hline & $\boldsymbol{B}_{i j}(\mathrm{eV})$ & $\boldsymbol{\rho}_{i j}(\AA)$ \\
\hline Er-O & 3771 & 0.28 \\
$\mathrm{O}-\mathrm{O}$ & 390 & 0.36 \\
\hline
\end{tabular}

vacuum, and $\rho$ is the softness parameter. The parameters used in the MD simulation are summarized in Table 1.

The simulations were carried out for a system of $2000 \mathrm{Er}$ and $3000 \mathrm{O}$ atoms in the unit cell with a random atomic configuration. The cell volume was determined from the number densities of $l$ - $\mathrm{Er}_{2} \mathrm{O}_{3}$ at the melting point, which were calculated with the density measured by the ISS-ELF. Periodic boundary conditions were used, and the longrange Coulomb interaction was treated with Ewald's summation. A time step of $1 \mathrm{fs}$ was used in the Verlet algorithm. First, the temperature of the system was maintained at $4000 \mathrm{~K}$ for 20,000 time steps and then cooled to $2923 \mathrm{~K}$ over 20,000 steps. The structural model was finally annealed at $2923 \mathrm{~K}$ for 150,000 steps. After the MD simulation, RMC refinement was conducted using the $\mathrm{RMC}++\operatorname{code}^{24}$. The benchmark RMC runs were performed using simulation boxes with $250,500,1000$, and 3000 particles.

\section{Density functional - molecular dynamics simulation}

The simulations based on the density functional theory (DFT) of the electronic structure were performed with the projector augmented wave (PAW) method ${ }^{25}$, implemented in the VASP software ${ }^{26,27}$. The PAW potentials supplied within VASP for $\operatorname{Er}(5 p, 5 d$, and $6 s$, with $114 f$ electrons frozen in the core) and $\mathrm{O}(2 s, 2 p)$ were tested and used (see supplementary information for a comparison between the frozen core approximation and the treating of the Er- $4 f$ electrons explicitly, Fig. S1). For a liquid sample of 500 atoms, the energy cutoff of the plane waves was set to $400 \mathrm{eV}$, with a single $\Gamma$-point in the Brillouin zone. In comparison, the bulk crystalline $(c)$ $\mathrm{Er}_{2} \mathrm{O}_{3}$ unit cell was fully relaxed until the forces on all atoms were below $0.01 \mathrm{eV} / \AA$ with a $\Gamma$-centered $2 \times 2 \times 2$ k-point grid and a plane-wave energy cutoff of $550 \mathrm{eV}$. The PBE functional ${ }^{28}$ was used for the geometry optimization and the molecular dynamics simulations, whereas the HSE06 hybrid functional ${ }^{29}$ was used to obtain the electronic densities of states (DOSs) and their projections to produce more realistic electronic band gaps and test the effect of the $4 f$ electrons in $c-\mathrm{Er}_{2} \mathrm{O}_{3}$. The effective charges and atomic volumes were evaluated by Bader analysis $^{30-32}$ using the PBE functional.

The density functional - molecular dynamics (DF-MD) simulations were performed with a Nóse-Hoover thermostat $^{33}$ and a time step of $2 \mathrm{fs}$, with an initial atomic configuration given by the benchmark RMC model mentioned above with 500 atoms. The system was simulated at $2923 \mathrm{~K}\left(\sim 2650^{\circ} \mathrm{C}\right)$ for a total of $30 \mathrm{ps}$, where the last 25 ps were used for data collection (Fig. S2). The electron occupancy was described with a Fermi smearing corresponding to the $k_{\mathrm{B}} T$ value at the target temperature. The mean-square displacements (MSDs) of the atoms show a liquid (diffusion) behavior where equilibrium is already achieved during the first few picoseconds.

\section{Topological analysis using a persistent homology}

The homology of atomic configurations has been investigated using the persistence diagram $D_{1}$, which consists of two-dimensional histograms showing a persistent homology. The details of the analysis are described elsewhere ${ }^{34}$. The persistence diagram $D_{1}$ of a set of atoms is given by the following thickening process of spheres: (1) place a sphere with a radius $r$ at the center of each atom, (2) increase the radii of the spheres from 0 to a sufficiently large value, and (3) encode the pair of birth and death radii $\left(b_{i}, d_{i}\right)$ for each ring $c_{i}$ consisting of a set of spheres. The persistence diagram is then constructed by the twodimensional histogram on the birth and death plane obtained by the pairs for independent $c_{i}, i=1, \ldots, K$. Here, the birth (death) radius is defined as the radius of spheres at which the ring $c_{i}$ first appears (disappears). The birth radius has information about the distances between atoms of the ring $c_{i}$, and the death radius has information about the size of the ring. The persistence diagram provides statistical information on the shapes of all independent rings and thereby provides insight into intermediate ordering in the liquid structure. The rings and cavities detected by this process are recorded for the computation of the persistence diagrams; hence, their geometric shapes can be identified for further analysis. The persistence diagrams were calculated using the HomCloud package ${ }^{35}$.

\section{Results and discussion \\ Density data}

Figure 1 shows the density of $l-\mathrm{Er}_{2} \mathrm{O}_{3}$ as a function of temperature, which exhibits a linear temperature dependence. The least-squares fit to the data is given by the following equation:

$$
\rho(T)=\rho_{\mathrm{m}}\left[1-\alpha\left(T-T_{\mathrm{m}}\right)\right]\left(\mathrm{kg} / \mathrm{m}^{3}\right),
$$

where $\rho_{\mathrm{m}}$ is the molten density at $T_{\mathrm{m}}\left(8170 \mathrm{~kg} / \mathrm{m}^{3}\right)$ and $\alpha$ $\left(=1 / \rho_{\mathrm{m}}[\mathrm{d} \rho(T) / \mathrm{d} T]\right)$ is the thermal expansion coefficient and is assumed to be constant $\left(1.0 \times 10^{-4} \mathrm{~K}^{-1}\right)$ at any temperature of the liquid. The correlation coefficient of this fitting is 0.98 . The uncertainty in the measurements is estimated to be $2 \%$ from the image resolution $(640 \times 480$ pixels) and from the uncertainty in the mass measurement $( \pm 0.1 \mathrm{mg})$. 


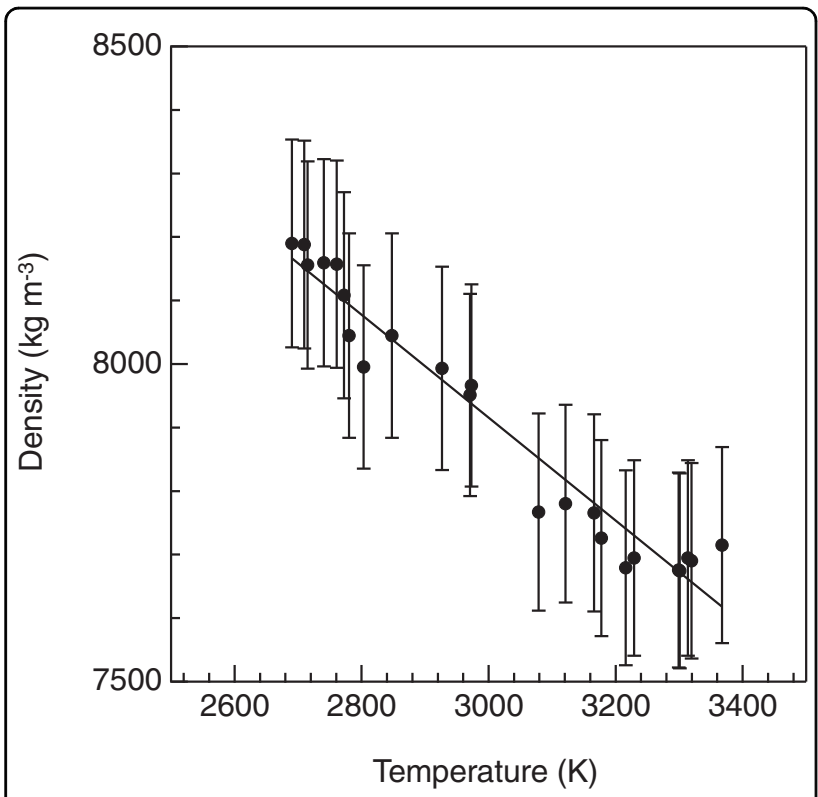

Fig. 1 Density of $\mathrm{I}-\mathrm{Er}_{2} \mathrm{O}_{3}$ as a function of temperature.

Table 2 Densities at $T_{m}$ and the thermal expansion coefficients.

\begin{tabular}{lllll}
\hline & $\mathbf{S i O}_{\mathbf{2}}{ }^{36}$ & $\mathbf{A l}_{\mathbf{2}} \mathbf{O}_{\mathbf{3}}{ }^{{ }^{37}}$ & $\mathbf{Z r O}_{\mathbf{2}}{ }^{18}$ & $\mathbf{E r}_{\mathbf{2}} \mathbf{O}_{\mathbf{3}}$ \\
\hline Density at $T_{\mathrm{m}}\left(\mathrm{kg} / \mathrm{m}^{3}\right)$ & 2140 & 2930 & 5050 & 8170 \\
$\begin{array}{l}\text { Thermal expansion coefficient } \\
\left(10^{-4} \mathrm{~K}^{-1}\right)\end{array}$ & 1.0 & 1.2 & 1.8 & 1.0 \\
\hline
\end{tabular}

The density and the expansion coefficient for $l-\mathrm{Er}_{2} \mathrm{O}_{3}$, together with those for $l-\mathrm{SiO}_{2}{ }^{36}$ and other non-GFLs ${ }^{18,37}$, are compared in Table 2. Although the density trends increase with increasing cation atomic number, they do not show a clear relation. On the other hand, the thermal expansion coefficients show a similarity as each value approaches $1 \times 10^{-4} \mathrm{~K}^{-1}$. The thermal expansion coefficient of $l-\mathrm{Er}_{2} \mathrm{O}_{3}$ is especially close to those of $l-\mathrm{SiO}_{2}$ and $l-\mathrm{Al}_{2} \mathrm{O}_{3}$.

\section{Structure factors and real-space functions}

The Faber-Ziman X-ray total structure factors, $S(Q)$, for $l-\mathrm{Er}_{2} \mathrm{O}_{3}, l-\mathrm{SiO}_{2}{ }^{38}, l-\mathrm{Al}_{2} \mathrm{O}_{3}{ }^{11}$, and $l-\mathrm{ZrO}_{2}{ }^{18}$, together with the results of the MD-RMC simulation for $l-\mathrm{Er}_{2} \mathrm{O}_{3}$, are compared in Fig. 2a. It is noted that the scattering vector $Q$ is scaled by multiplying by $r_{\mathrm{A}-\mathrm{X}}$ (distance between the center and corners of the polyhedron). The experimental $S(Q)$ of $l$ - $\mathrm{Er}_{2} \mathrm{O}_{3}$ (solid cyan curve) is well reproduced by the MDRMC simulation (dotted black curve) using the liquid density measured by the ISS-ELF shown in Fig. 1. A welldefined first sharp diffraction peak (FSDP $)^{39}$ is observed only for $l-\mathrm{SiO}_{2}(\mathrm{GFL})$ at $Q r_{\mathrm{A}-\mathrm{X}}=2.6$, while a principal peak $(\mathrm{PP})^{39}$ is observed in both the $l-\mathrm{ZrO}_{2}$ and $l-\mathrm{Er}_{2} \mathrm{O}_{3}$ data at
$Q r_{\mathrm{A}-\mathrm{X}} \sim 4.5$. On the other hand, $l-\mathrm{Al}_{2} \mathrm{O}_{3}$ gives rise to a small peak between the FSDP and PP, suggesting that the structure of $l-\mathrm{Al}_{2} \mathrm{O}_{3}$ is intermediate ${ }^{17}$ between $l-\mathrm{SiO}_{2}$ and $l-\mathrm{ZrO}_{2} / l-\mathrm{Er}_{2} \mathrm{O}_{3}$. It is well known that the PP reflects the packing of oxygen atoms in neutron diffraction data ${ }^{40}$, since neutrons are sensitive to oxygen. For the same reason, a PP is not observed in the $\mathrm{X}$-ray $S(Q)$ for $l$ - $\mathrm{SiO}_{2}$ (see Fig. 2a), and the origin of the PP in $l-\mathrm{ZrO}_{2}$ and $l-\mathrm{Er}_{2} \mathrm{O}_{3}$ is ascribed to the packing of cations. The $\mathrm{X}$-ray total correlation functions $T$ (r) for $l-\mathrm{Er}_{2} \mathrm{O}_{3}, l-\mathrm{SiO}_{2}{ }^{38}, l-\mathrm{Al}_{2} \mathrm{O}_{3}{ }^{11}$, and $l-\mathrm{ZrO}_{2}{ }^{18}$ are shown in Fig. 2b. The first peak observed at approximately $2.2 \AA$ is assigned to the Er-O correlation, and a tail to $\sim 3 \AA$ implies the formation of distorted ErOn polyhedra in the liquid. The second peak, observed at $3.7 \AA$, can be assigned mainly to the Er-Er correlation, and the O-O correlation peak is unclear due to its small weighting factor for X-rays. The Er-O correlation length of $2.2 \AA$, as well as that of $\mathrm{Zr}-\mathrm{O}$ $(2.1 \AA)$, is longer than those of $\mathrm{Si}-\mathrm{O}(\sim 1.63 \AA$ at $2373 \mathrm{~K})$ and $\mathrm{Al}-\mathrm{O}(\sim 1.78 \AA$ at $2400 \mathrm{~K})$ owing to substantial differences between the ionic radii of the elements. The increased cation-oxygen correlation length in the liquid phases of $\mathrm{Er}-\mathrm{O}$ and $\mathrm{Zr}-\mathrm{O}$ suggests that the oxygen coordination number around cations is higher than 4 because the $\mathrm{Er}-\mathrm{O}$ correlation length ( $2.2 \AA$ ) or $\mathrm{Zr}-\mathrm{O}$ correlation length $(2.1 \AA$ ) is close to the sum of the ionic radii ${ }^{41}$ of oxygen $(1.35 \AA)$ and six-fold erbium $(0.89 \AA)$ or zirconium $(0.72 \AA)$, respectively. The structures of $l-\mathrm{Er}_{2} \mathrm{O}_{3}$ and $l-\mathrm{ZrO}_{2}$ therefore consist of large interconnected polyhedral units and are very different from those of $l-\mathrm{SiO}_{2}$ and $l-\mathrm{Al}_{2} \mathrm{O}_{3}$. This behavior is consistent with the fact that the peaks observed at $\mathrm{Qr}_{\mathrm{A}-\mathrm{X}} \sim 4.5$ in Fig. 2a are not the FSDP, which is typically associated with intermediate-range ordering in oxide glasses and liquids; thus, there is no such ordering in $l-\mathrm{Er}_{2} \mathrm{O}_{3}$ and $l-\mathrm{ZrO}_{2}$ due to a very densely packed structure.

\section{Coordination number distributions from the simulation}

The coordination number distributions, $N_{\mathrm{A}-\mathrm{X}}$ and $N_{\mathrm{X}-\mathrm{A}}$, for $l-\mathrm{Er}_{2} \mathrm{O}_{3}, l-\mathrm{SiO}_{2}{ }^{42}, l-\mathrm{Al}_{2} \mathrm{O}_{3}{ }^{11}$, and $l-\mathrm{ZrO}_{2}{ }^{18}$ obtained from the simulation are compared in Fig. 3a, b, and their average values are summarized in Table 3 . The $\mathrm{Er}-\mathrm{O}$ coordination number (up to $3.0 \AA$ ) is found to be 6.1 from our combined MD-RMC simulation, which is rather close to the crystalline phase ${ }^{43}$, and the $\mathrm{O}-\mathrm{Er}$ coordination number can be estimated to be 4.1. It is suggested that the cations are tetrahedrally coordinated in $l-\mathrm{SiO}_{2}$ (GFL), while they are octahedrally coordinated in $l-\mathrm{ZrO}_{2}$ and $l-\mathrm{Er}_{2} \mathrm{O}_{3}$ (non-GFLs), and the cation-oxygen coordination number in $l-\mathrm{Al}_{2} \mathrm{O}_{3}$ is intermediate ${ }^{17}$ between GFL and $l-\mathrm{ZrO}_{2} / l$ $\mathrm{Er}_{2} \mathrm{O}_{3}$, although $l-\mathrm{Al}_{2} \mathrm{O}_{3}$ is a non-GFL. This behavior is consistent with that of the first correlation peaks in experimental real-space functions (see Fig. $2 \mathrm{~b}$ ) and with the fact that the viscosity of $l-\mathrm{ZrO}_{2}$ is approximately one-tenth of that in $l-\mathrm{Al}_{2} \mathrm{O}_{3}{ }^{18}$. Another interesting behavior is observed for the oxygen-cation coordination numbers. It is 
a

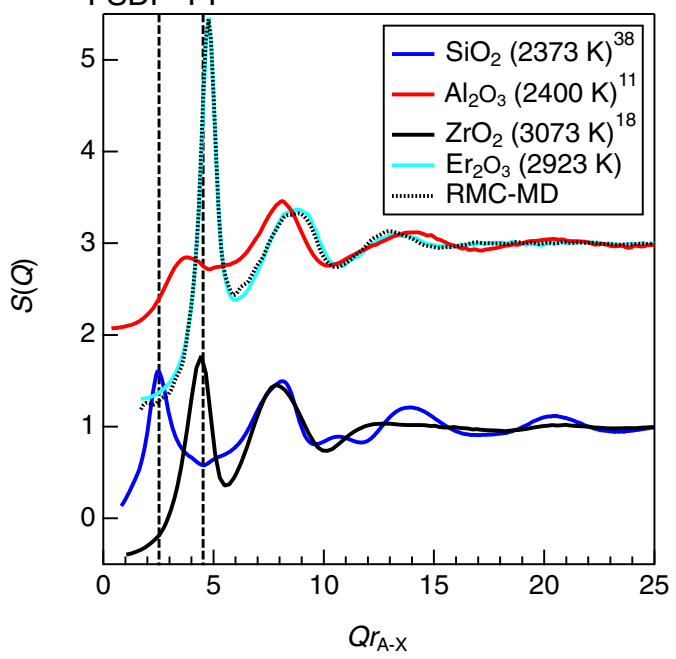

b

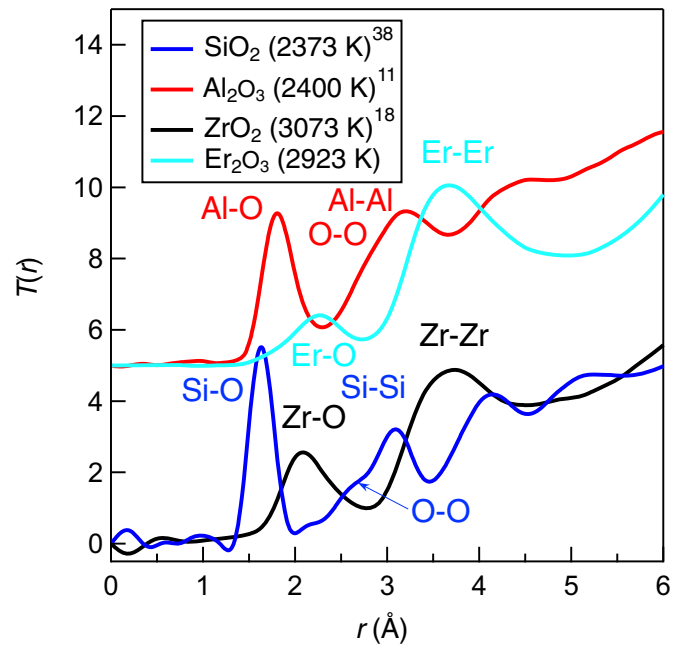

Fig. 2 High-energy X-ray diffraction data for high-temperature oxide liquids. a Faber-Ziman X-ray total structure factors, $S(Q)$, for $I$ - $E r_{2} \mathrm{O}_{3}$, $1-\mathrm{SiO}_{2}{ }^{38}, I-\mathrm{Al}_{2} \mathrm{O}_{3}{ }^{11}$, and $1-\mathrm{ZrO}_{2}{ }^{18}$ together with that of $\mathrm{I}-\mathrm{Er}_{2} \mathrm{O}_{3}$ derived from the MD-RMC simulation. The $\mathrm{I}-\mathrm{Er}_{2} \mathrm{O}_{3}$ and $l-\mathrm{Al}_{2} \mathrm{O}_{3}$ data are displaced upward by 2 for clarity. b Total correlation functions, $T(r)$, for $l-\mathrm{SiO}_{2}{ }^{38}, I-\mathrm{Al}_{2} \mathrm{O}_{3}{ }^{11}$, and $\mathrm{l}-\mathrm{ZrO}_{2}{ }^{18}$. The $l-\mathrm{Er}_{2} \mathrm{O}_{3}$ and $\mathrm{Al}_{2} \mathrm{O}_{3}$ data are displaced upward by 5 for clarity.
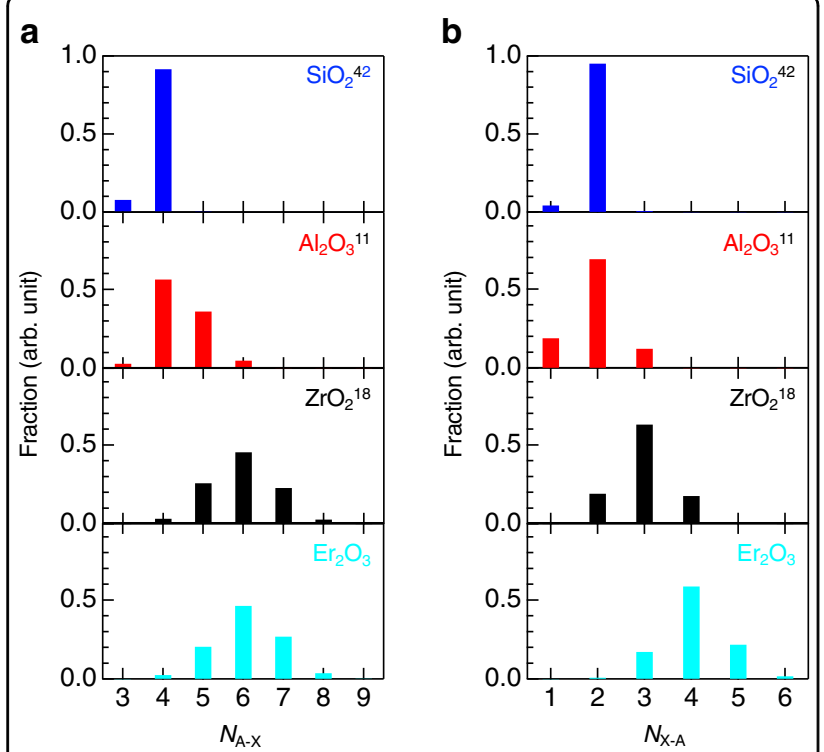

Fig. 3 Coordination number distributions in $\mathrm{I}-\mathrm{Er}_{2} \mathrm{O}_{3}, \mathrm{I}-\mathrm{SiO}_{2}{ }^{42}, \mathrm{I}$ $\mathrm{Al}_{2} \mathrm{O}_{3}{ }^{11}$, and $l-\mathrm{ZrO}_{2}{ }^{18}$. a $N_{\mathrm{A}-\mathrm{X}}, \mathrm{b} N_{\mathrm{X}-\mathrm{A}}$.

demonstrated that oxygen is twofold in $l-\mathrm{SiO}_{2}$, which is a signature of the formation of a sparse network, while triclusters $\left(\mathrm{XA}_{3}\right)$ are dominant in $l-\mathrm{Al}_{2} \mathrm{O}_{3}$ and $l-\mathrm{ZrO}_{2}$. The formation of tetraclusters $\left(\mathrm{XA}_{4}\right)$ is confirmed in $l-\mathrm{Er}_{2} \mathrm{O}_{3}$, suggesting that this behavior is a distinct feature of this liquid. We suggest that the behavior of the coordination numbers in a series of oxide liquids is affected by both the composition and the ionic radii between the constituent anions and cations. For instance, the ionic radii of $\mathrm{Si}$ and $\mathrm{Al}$
Table 3 Average coordination numbers derived from the simulations.

\begin{tabular}{lllll}
\hline & $\begin{array}{l}\mathrm{SiO}_{2}{ }^{42} \\
(\mathbf{2 2 7 3} \mathbf{K})\end{array}$ & $\begin{array}{l}\mathrm{Al}_{2} \mathrm{O}_{3}{ }^{11} \\
(\mathbf{2 4 0 0 ~ K )}\end{array}$ & $\begin{array}{l}\mathrm{ZrO}_{2}{ }^{18} \\
(\mathbf{3 0 7 3} \mathbf{K})\end{array}$ & $\begin{array}{l}\mathrm{Er}_{2} \mathrm{O}_{3} \\
\mathbf{( 2 9 2 3 ~ K )}\end{array}$ \\
\hline$N_{\mathrm{A}-\mathrm{X}}$ & 3.9 & 4.4 & 6.0 & 6.1 \\
$N_{X-\mathrm{A}}$ & 2.0 & 2.9 & 3.0 & 4.1 \\
\hline
\end{tabular}

are small, which results in tetrahedral coordination, although the Al-O coordination number is greater than four on average. The tetracluster formation is caused by the ratio of $\mathrm{Er}$ and $\mathrm{O}$ in $\mathrm{Er}_{2} \mathrm{O}_{3}$.

\section{Very sharp principal peak (PP) in $/-\mathrm{Er}_{2} \mathrm{O}_{3}$}

As shown in Fig. 2a, the PP of $l-\mathrm{Er}_{2} \mathrm{O}_{3}$ is very sharp compared to that of $l-\mathrm{ZrO}_{2}$. The FWHM of the PP in $l$ $\mathrm{Er}_{2} \mathrm{O}_{3}$ is 0.4299 , in comparison to 0.7669 in $l-\mathrm{ZrO}_{2}$ (see Fig. 4). A simulation box with 501 particles was used in the previous RMC - density functional (DF) simulation for $l-\mathrm{ZrO}_{2}{ }^{18}$, where a good agreement was observed between the experimental data and simulation (see Fig. $4 a)$. However, as can be seen in the inset data of Fig. 4b, a simulation box of 500 particles is insufficient to reproduce the sharp PP in $l-\mathrm{Er}_{2} \mathrm{O}_{3}$; larger atomic models are needed to reproduce this feature. Insight into the structure of $l$ $\mathrm{Er}_{2} \mathrm{O}_{3}$, in comparison with those of $l-\mathrm{SiO}_{2}$ and other nonGFLs, can be obtained by calculating the Faber-Ziman partial structure factors, $S_{i j}(Q)$, and the Bhatia-Thornton ${ }^{44}$ number - number partial structure factor, $S_{\mathrm{NN}}(Q)$, which 
a

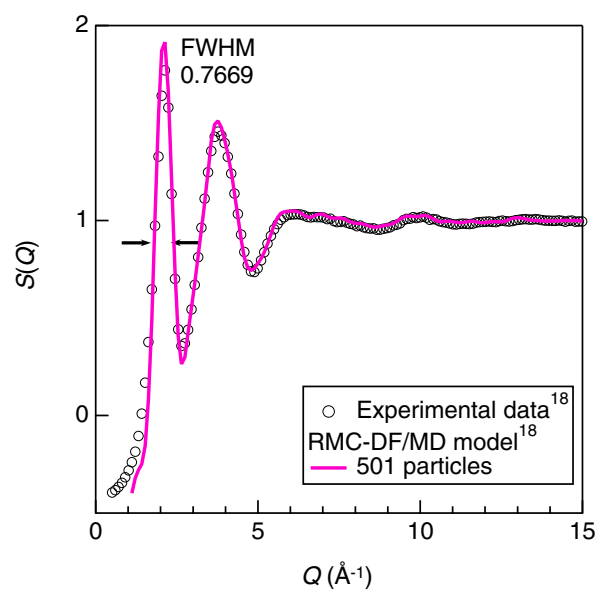

b

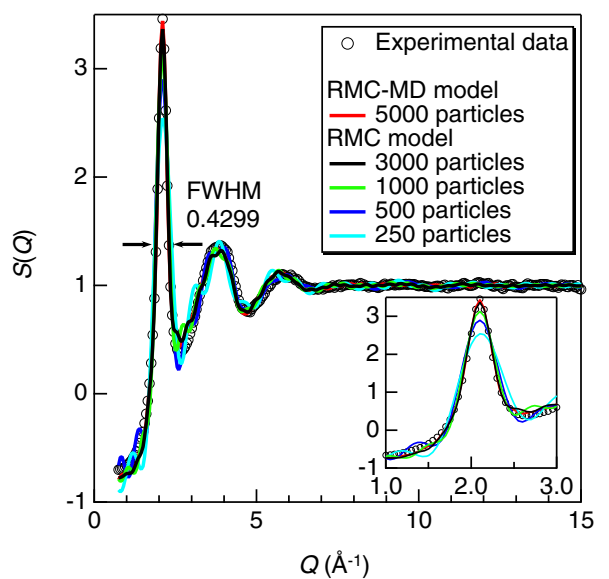

Fig. 4 X-ray total structure factors, $S(Q)$, obtained from high-energy X-ray diffraction measurements and simulations. a $/-\mathrm{ZrO}_{2}{ }^{18}, \mathbf{b} /-\mathrm{Er}_{2} \mathrm{O}_{3}$. The inset shows an enlarged principal peak.

indicates the topological order in a system:

$$
S_{\mathrm{NN}}(Q)=c_{\mathrm{A}}^{2} S_{\mathrm{AA}}(Q)+c_{\mathrm{X}}^{2} S_{\mathrm{XX}}(Q)+2 c_{\mathrm{A}} c_{\mathrm{X}} S_{\mathrm{AX}}(Q),
$$

where $S_{i j}(Q)$ is a Faber-Ziman partial structure factor and $c_{i}$ denotes the atomic fraction of chemical species $i$. Moreover, it is possible to compare data for the four liquids while ignoring the difference in the sensitivity of elements to X-rays because the weighting factors for Xrays are eliminated in $S_{\mathrm{NN}}(Q)$. The $S_{i j}(Q)$ values calculated from the simulation models for $l-\mathrm{Er}_{2} \mathrm{O}_{3}, l-\mathrm{SiO}_{2}{ }^{42}, l$ $\mathrm{Al}_{2} \mathrm{O}_{3}{ }^{11}$, and $l-\mathrm{ZrO}_{2}{ }^{18}$ are shown in Fig. 5a. It is confirmed that a very sharp PP in $l-\mathrm{Er}_{2} \mathrm{O}_{3}$ can be assigned to the ErEr correlation. The $S_{\mathrm{NN}}(Q)$ for $l-\mathrm{Er}_{2} \mathrm{O}_{3}$ and those for $l$ $\mathrm{SiO}_{2}$ and other non-GFLs are compared in Fig. 5b. As mentioned above, only $l-\mathrm{SiO}_{2}$ exhibits an FSDP at $Q r_{\mathrm{A}-\mathrm{X}}$ $=2.6$. The $Q_{\mathrm{FSDP}}$ position arises from an underlying periodicity of $2 \pi / Q_{F S D P}$ that originates, for example, from the formation of pseudo-Bragg planes with a finite correlation length of $2 \pi / \Delta Q_{\mathrm{FSDP}}$ in $l-\mathrm{SiO}_{2}$, while neither $l-\mathrm{Al}_{2} \mathrm{O}_{3}, l-\mathrm{ZrO}_{2}$, nor $l-\mathrm{Er}_{2} \mathrm{O}_{3}$ show an FSDP in $S_{\mathrm{NN}}(Q)$, as discussed in Kohara et al. ${ }^{18}$. Since the Bhatia-Thornton $S_{\mathrm{NN}}(Q)$ can eliminate the weighting factors for X-rays, the absence of an FSDP in $S_{\mathrm{NN}}(Q)$ is characteristic of a nonGFL. Another important feature in $S_{\mathrm{NN}}(Q)$ is that $l-\mathrm{SiO}_{2}$ and $l-\mathrm{Al}_{2} \mathrm{O}_{3}$ exhibit a second PP at $Q r_{\mathrm{A}-\mathrm{X}} \sim 5$, while a PP is not distinct in the $l-\mathrm{ZrO}_{2}$ or $l-\mathrm{Er}_{2} \mathrm{O}_{3}$ data.

The absence of an FSDP in the $l-\mathrm{ZrO}_{2}$ and $l-\mathrm{Er}_{2} \mathrm{O}_{3}$ data suggests that both cations and oxygen are densely packed. To confirm this in real space for $l-\mathrm{Er}_{2} \mathrm{O}_{3}$, the partial pair distribution functions, $g_{i j}(r)$, of $l-\mathrm{Er}_{2} \mathrm{O}_{3}$ are compared with those of $l-\mathrm{SiO}_{2}$ in Fig. 6a. The atomic distance $r$ is scaled by dividing by $r_{\mathrm{A}-\mathrm{X}}$ (distance between the center and corners of the polyhedron). It is found that the scaled first A-A and $\mathrm{X}-\mathrm{X}$ correlation distances of $l-\mathrm{Er}_{2} \mathrm{O}_{3}$ are much shorter than those of $l-\mathrm{SiO}_{2}$, demonstrating that $l-\mathrm{Er}_{2} \mathrm{O}_{3}$ has a much more densely packed structure, manifested by the formation of the $\mathrm{OEr}_{4}$ tetracluster network shown in Fig. 6b. This network cannot be found in $l-\mathrm{Al}_{2} \mathrm{O}_{3}$ nor in $l-\mathrm{ZrO}_{2}$, suggesting that the very sharp PP in $l-\mathrm{Er}_{2} \mathrm{O}_{3}$ is a specific signature of the formation of a tetracluster network with long-range periodicity.

\section{Topology and homology in $I-\mathrm{Er}_{2} \mathrm{O}_{3}$}

To reveal the origin of the very sharp PP in $l-\mathrm{Er}_{2} \mathrm{O}_{3}$, we calculated the bond angle distributions of the liquid and crystal $^{43}$ and summarized them in Fig. 7. A pronounced difference was found between the liquid and crystal data for the $\mathrm{O}-\mathrm{Er}-\mathrm{O}$ and $\mathrm{Er}-\mathrm{O}-\mathrm{Er}$ distributions. The $\mathrm{O}-\mathrm{Er}-\mathrm{O}$ bond angle distribution exhibits two peaks at $80^{\circ}$ and $140^{\circ}$, suggesting that $\mathrm{ErO}_{6}$ polyhedra are highly distorted in the liquid. Another interesting feature is that the Er-O-Er bond angle distribution exhibits a peak at $\sim 180^{\circ}$ in addition to the peak at $\sim 90^{\circ}$, which is not observed for the crystal ${ }^{43}$ nor in $l-\mathrm{ZrO}_{2}{ }^{18}$. This two-peak structure in the Er-O-Er bond angle distribution indicates the formation of a distorted $\mathrm{OEr}_{4}$ tetracluster network, whereas tetraclusters are symmetric (comprising regular tetrahedra) in the crystalline phase. This behavior suggests that the coordination of $\mathrm{OEr}_{4}$ tetraclusters is more octahedral-like and hence tolerant of disorder even in the liquid due to the distortion, providing a linear arrangement manifested by a prominent peak observed at $180^{\circ}$ in the Er-O-Er bond angle distribution. This is clearly visible in Fig. 6c, where linear atomic arrangements are highlighted by the magenta lines. 
a

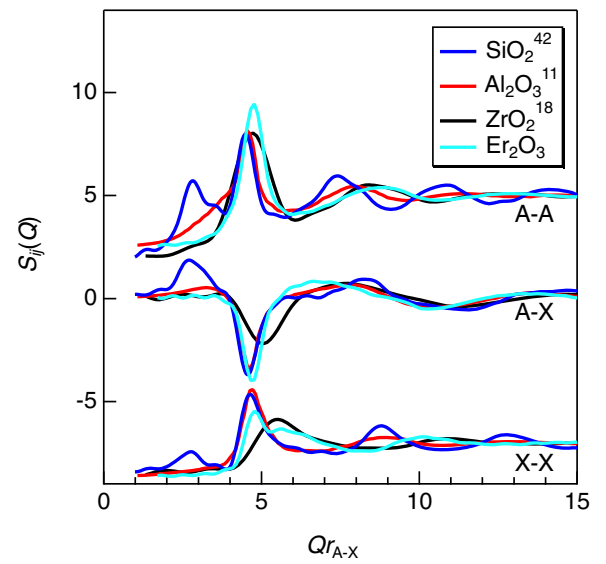

b

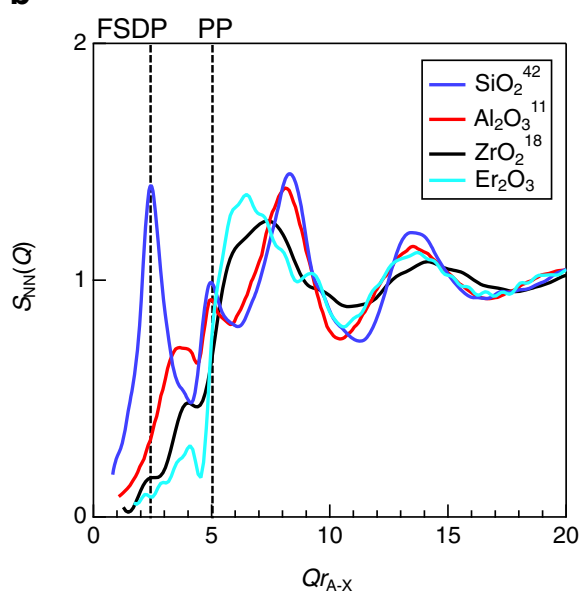

Fig. 5 Partial structure factors for $I-\mathrm{Er}_{2} \mathrm{O}_{3}, \mathrm{I}-\mathrm{SiO}_{2}{ }^{42}, \mathrm{I}-\mathrm{Al}_{2} \mathrm{O}_{3}{ }^{11}$, and $\mathrm{I}-\mathrm{ZrO}_{2}{ }^{18}$. a Faber-Ziman partial structure factors. $\mathbf{b}$ Bhatia-Thornton number number partial structure factors, $S_{N N}(Q)$. The scattering vector $Q$ is scaled by multiplying by $r_{A-x}$ (distance between the center and corner of the polyhedron).

a

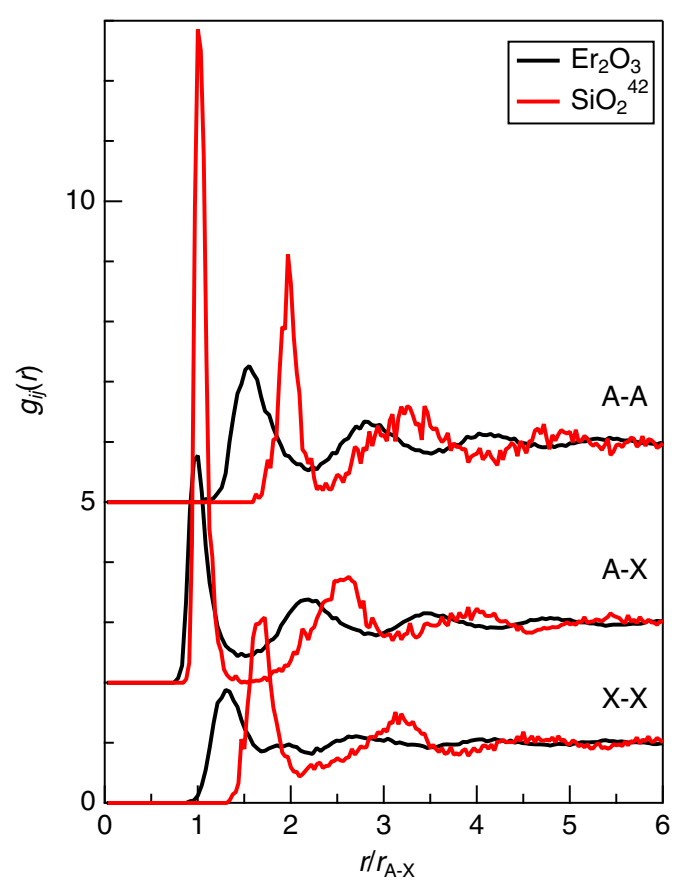

b

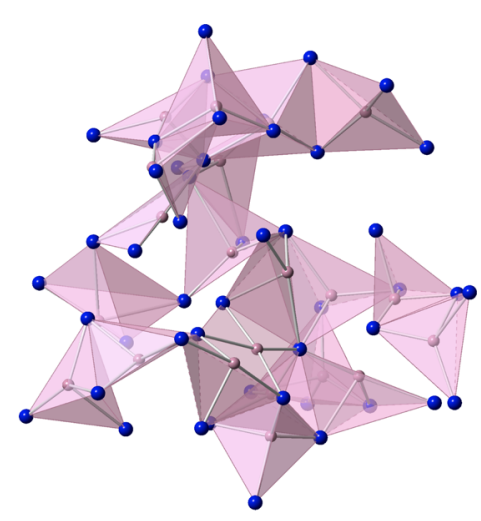

C

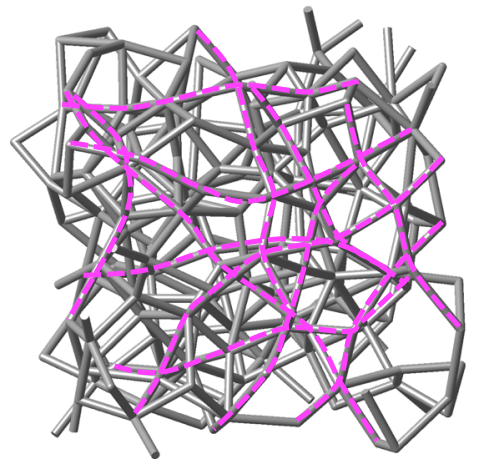

Fig. 6 Analyses in real space. a Partial pair-distribution functions, $g_{i j}(r)$, for $l-\mathrm{Er}_{2} \mathrm{O}_{3}$ and $l-\mathrm{SiO}_{2}{ }^{42}$. b Visualization of the $\mathrm{OEr}_{4}$ tetracluster network in $\mathrm{I}-\mathrm{Er}_{2} \mathrm{O}_{3}$. Pink, oxygen; blue, erbium. c Visualization of the nearly linear arrangements of Er-O-Er.

To shed light on the similarity in topology between the crystal and liquid phases, we calculated the persistence diagram for $l$ - $\mathrm{Er}_{2} \mathrm{O}_{3}$ and compared it with the crystal data in Fig. 8. The figures show the similarity between the crystal $^{43}$ and liquid phases. In particular, both the
Er-centric and O-centric persistence diagrams for $l$ - $\mathrm{Er}_{2} \mathrm{O}_{3}$ do not show a vertical profile along the death axis, which is a pronounced feature in a typical GFL, such as $l-\mathrm{SiO}_{2}{ }^{42}$. The short lifetime of the profile manifested by the small death value demonstrates that both the crystal and liquid 

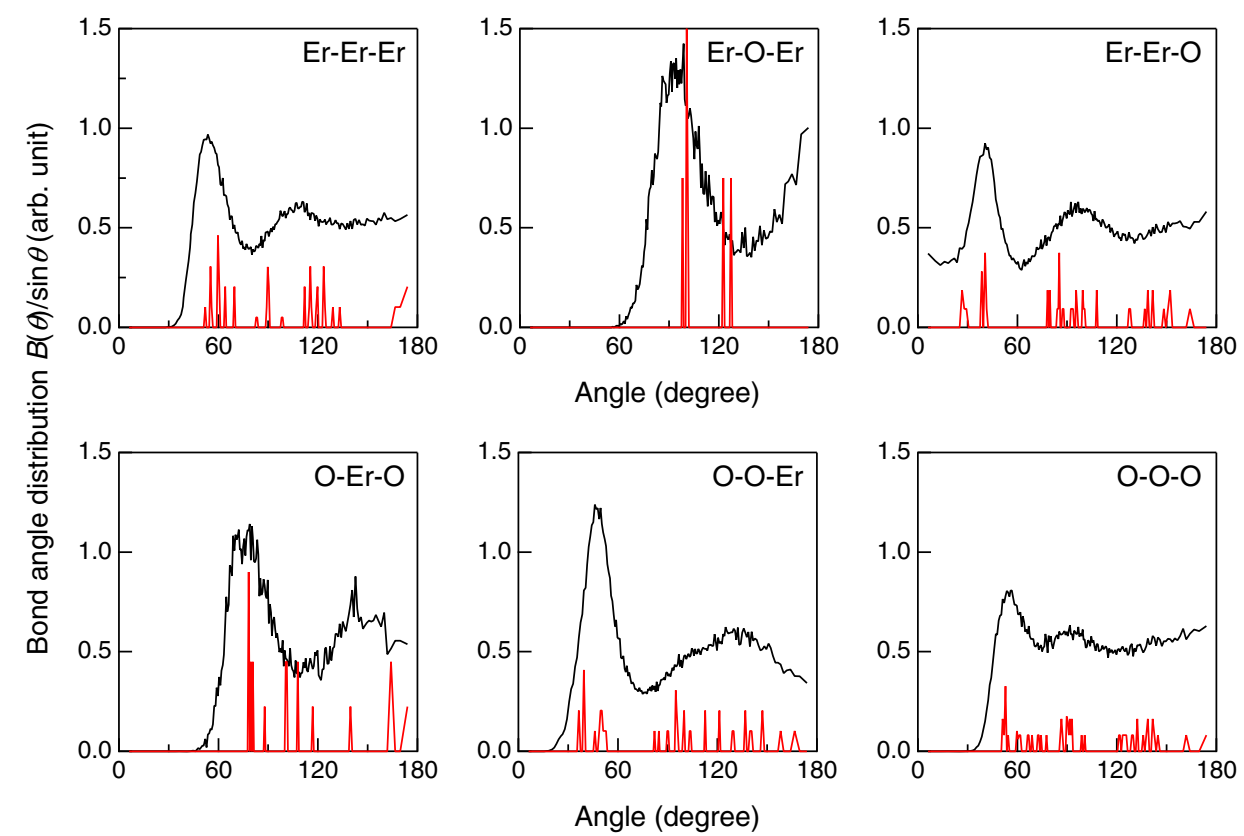

Fig. 7 Bond angle distributions for $\mathrm{Er}_{2} \mathrm{O}_{3}$. Black line, liquid; red line, crystal ${ }^{43}$. The $\mathrm{B}(\theta) / \sin \theta$ data for the crystalline data have been scaled by a factor of 20 for clarity.
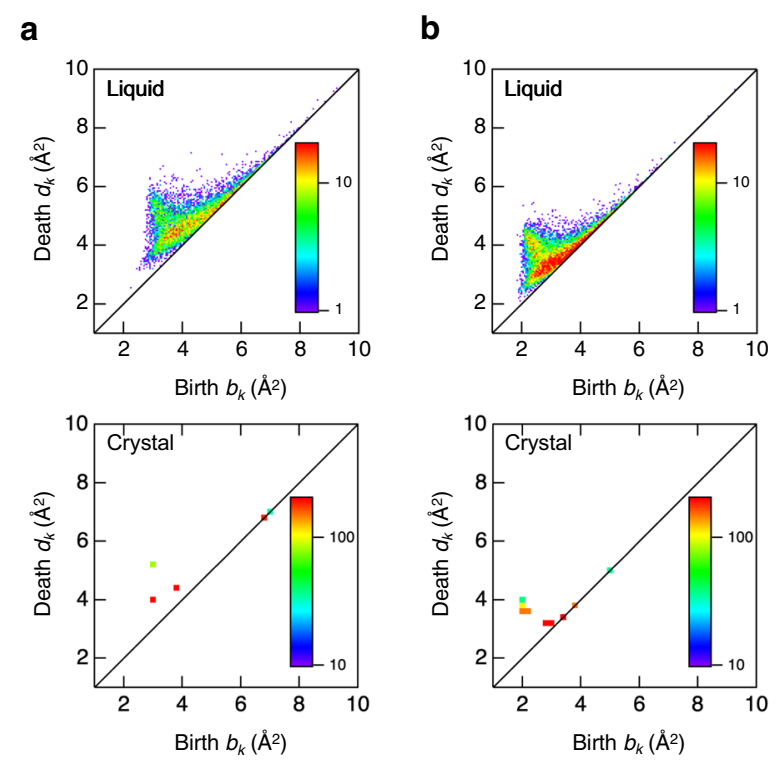

Fig. 8 Persistence diagrams of $\mathrm{Er}_{2} \mathrm{O}_{3}$. a Er-centric persistence diagram, b O-centric persistence diagram.

phases exhibit a very densely packed structure associated with the formation of tetraclusters in both phases. We suggest that this similarity is a signature of non-GFL behavior.

\section{Electronic structure and viscosity of $I-\mathrm{Er}_{2} \mathrm{O}_{3}$}

As previously mentioned, a 500-atom RMC model of $l$ - $\mathrm{Er}_{2} \mathrm{O}_{3}$ appeared to be too small in reproducing the very sharp PP accurately (see Fig. S3). Nevertheless, we used this model as a starting structure in our DF-MD simulations to study the electronic structure and atomic diffusion in the liquid phase. The electronic density of states (DOS) and effective charges were calculated for snapshots of $l-\mathrm{Er}_{2} \mathrm{O}_{3}$ atomic structures and a fully DFTrelaxed $(0 \mathrm{~K}) c-\mathrm{Er}_{2} \mathrm{O}_{3}$ unit cell. The DOSs of $l$ - and $c$ $\mathrm{Er}_{2} \mathrm{O}_{3}$ are shown in Fig. 9a, b. The valence band consists mainly of $\mathrm{O}-2 p$ states, while the conduction band consists mainly of Er-5d states. As a measure of the orbital localization, we also present the inverse participation ratios (IPRs) for $l$ - $\mathrm{Er}_{2} \mathrm{O}_{3}$. The IPRs show increased weight at the valence band maximum (VBM), indicating stronger localization, whereas the states around the conduction band minimum (CBM) are delocalized. The obvious broadening of the valence and conduction bands in $l-\mathrm{Er}_{2} \mathrm{O}_{3}$ is caused by the distortion of the $\mathrm{ErO}_{n}$ polyhedra at elevated temperatures. The DOS for $l$ - $\mathrm{Er}_{2} \mathrm{O}_{3}$ reveals a band gap of $0.57 \mathrm{eV}$ in comparison to the substantially large band gap of $5.46 \mathrm{eV}$ in $c-\mathrm{Er}_{2} \mathrm{O}_{3}$. Previously, a vanishing band gap was reported for $l-\mathrm{ZrO}_{2}{ }^{18}$, but we ascribe this effect to the PBE functional, which is known to underestimate the real value, whereas the hybrid HSE06 functional used here predicts a more correct band gap. 


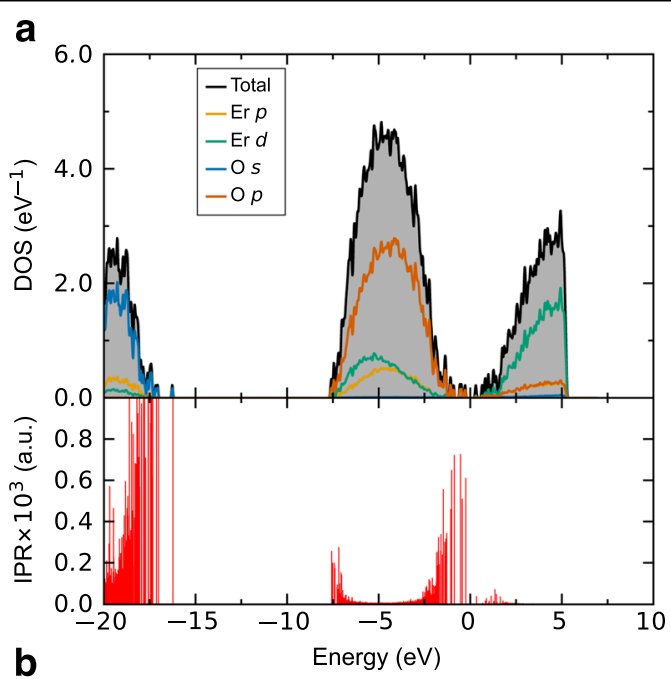

C
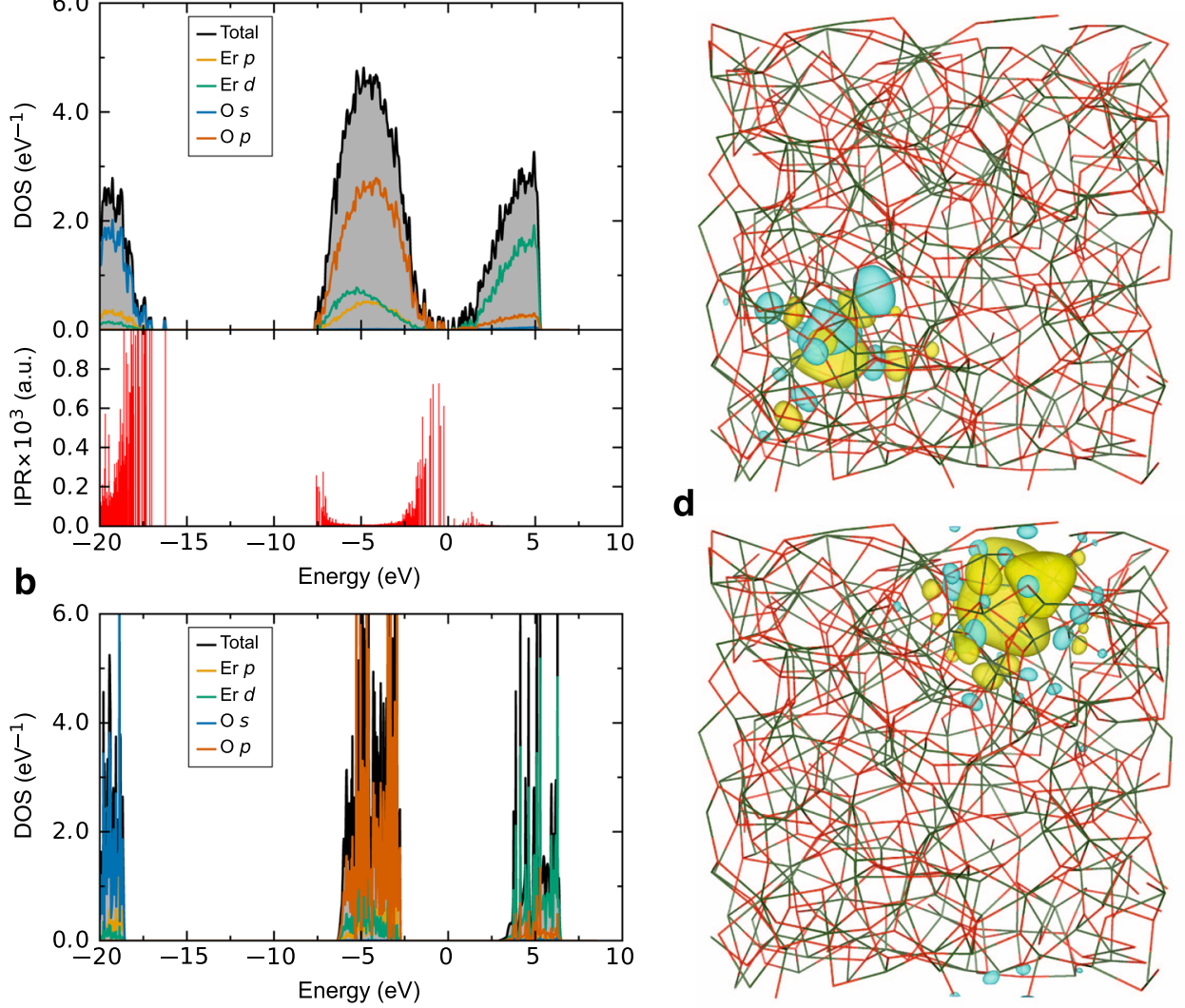

d

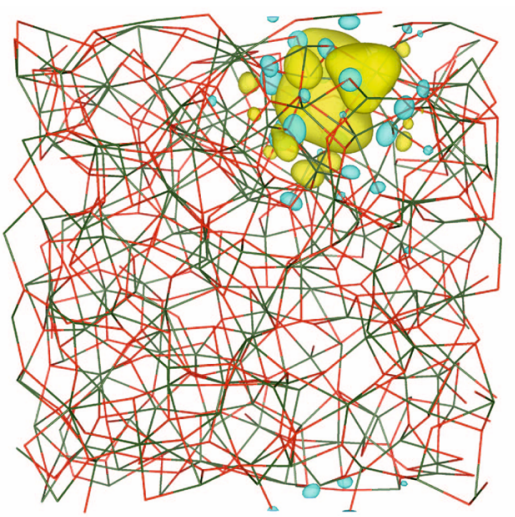

Fig. 9 The electronic structure of $\mathrm{Er}_{2} \mathrm{O}_{3}$. a Calculated atom-resolved DOS (top) and IPR (bottom) for $1-\mathrm{Er}_{2} \mathrm{O}_{3}$. $\mathbf{b}$ Calculated DOS of $c$ - $\mathrm{Er}_{2} \mathrm{O}_{3}$ for comparison. c Visualization of the highest occupied molecular band (HOMO). d Visualization of the lowest unoccupied molecular band (LUMO). Er and $\mathrm{O}$ atoms are shown in green and red, respectively. Yellow and cyan correspond to different signs of the wavefunction.

The difference in electronegativities of $\mathrm{Er}(1.24)$ and $\mathrm{O}$ (3.44) suggests predominantly ionic chemical bonding between the two atoms. This becomes clear from the partial DOS in Fig. 9a, and the calculated atomic charges are summarized in Table 4. The effective charges for $l-\mathrm{Er}_{2} \mathrm{O}_{3}$ are $+1.96 e$ and $-1.31 e$ for $\mathrm{Er}$ and $\mathrm{O}$, respectively, similar to those in $c-\mathrm{Er}_{2} \mathrm{O}_{3}$ (see Table 4). These values are in agreement with previous works on $l-\mathrm{ZrO}_{2}{ }^{18}$, glassy $\mathrm{MgO}-\mathrm{SiO}_{2}{ }^{45}$, and $\mathrm{CaO}-\mathrm{Al}_{2} \mathrm{O}_{3}{ }^{46}$ and are consistent with the nominal charges $\mathrm{Er}^{3+}$ and $\mathrm{O}^{2-}$ (systematically scaled down by a factor of $\left.\sim \frac{2}{3}\right)$. As for $\mathrm{ZrO}_{2}{ }^{18}$, the increased atomic volume of oxygen in the transition from $c$ - to $l-\mathrm{Er}_{2} \mathrm{O}_{3}$ compensates for the corresponding decreased oxygen coordination, which results in similar atomic charges for the two phases. Note here also the similarity with the charges used in the classic MD force field $(+2.1$ and $-1.4 e$ ).

A real space visualization of the highest occupied molecular band (HOMO) is shown in Fig. 9c, where the orbital is found to be distributed over a group of atoms. Locally, the shape of the HOMO is similar to that of $c$ - $\mathrm{Er}_{2} \mathrm{O}_{3}$ (Fig. S4); however, some deviations occur caused
Table 4 Average atomic charges and volumes calculated using the Bader method.

\begin{tabular}{llllll}
\hline System & Er & & & \multicolumn{1}{l}{$\mathbf{0}$} \\
\cline { 2 - 3 } \cline { 5 - 6 } & $\boldsymbol{Q}_{\text {eff }}(\boldsymbol{e})$ & $\boldsymbol{V}_{\text {at }}\left(\AA^{3}\right)$ & & $\boldsymbol{Q}_{\text {eff }}(\boldsymbol{e})$ & $\boldsymbol{V}_{\text {at }}\left(\AA^{3}\right)$ \\
\hline$C-\mathrm{Er}_{2} \mathrm{O}_{3}$ & +2.03 & 13.77 & & -1.35 & 15.22 \\
$1-\mathrm{Er}_{2} \mathrm{O}_{3}$ & +1.96 & 15.25 & & -1.31 & 16.25 \\
\hline
\end{tabular}

by the aforementioned distorted tetracluster network in $l$ - $\mathrm{Er}_{2} \mathrm{O}_{3}$. Figure 9d shows a real space visualization of the lowest unoccupied molecular band (LUMO), where most of the orbital is distributed over nonbonding regions inbetween the $\mathrm{ErO}_{n}$ polyhedra. The orbital projections for each of the bands reveal that the HOMO band shows mainly an O- $2 p$ character, while the LUMO band consists mainly of Er-5d states and some Er-5p character, in agreement with the DOS and IPR in Fig. 9a.

The DF-MD simulations above the melting point at $2650^{\circ} \mathrm{C}$ show that the atoms move rapidly, breaking old and forming new Er-O bonds on a picosecond time scale. 
The MSD analysis (Fig. S2) shows a consistent linear behavior as a function of time, the evaluated self-diffusion constants are $2.40 \times 10^{-5}$ and $5.84 \times 10^{-5} \mathrm{~cm}^{2} / \mathrm{s}$ for $\mathrm{Er}$ and $\mathrm{O}$, respectively, and the average self-diffusion constant is $4.64 \times 10^{-5} \mathrm{~cm}^{2} / \mathrm{s}$. By using the average value and assuming spherical particles in the Stoke-Einstein relation, one can estimate the viscosity, and the obtained value is $\sim 3 \times 10^{-3} \mathrm{~Pa} \mathrm{~s}^{-1}$ for $l-\mathrm{Er}_{2} \mathrm{O}_{3}$ in comparison to the previously reported value for $l-\mathrm{ZrO}_{2}$, i.e., $\sim 2 \times 10^{-3} \mathrm{~Pa} \mathrm{~s}^{-1}$ at $2800{ }^{\circ} \mathrm{C}^{18}$. Since these values are an order of magnitude smaller than that for $l-\mathrm{Al}_{2} \mathrm{O}_{3}$ (fragile liquid) and 9-10 orders of magnitude smaller than that for $l-\mathrm{SiO}_{2}$ (strong liquid), we characterize $l$ - $\mathrm{Er}_{2} \mathrm{O}_{3}$ as an "extremely fragile" liquid ${ }^{18}$.

We combined an aerodynamic levitation technique and a synchrotron high-energy X-ray diffraction and density measurement at the ISS for $l-\mathrm{Er}_{2} \mathrm{O}_{3}$ to reveal the structure of $\mathrm{A}_{2} \mathrm{X}_{3}$-type non-GFLs. As the main finding, we observed a very sharp PP in the diffraction data. The Er-O coordination number was estimated to be 6.1 from a combined MD-RMC simulation, which is comparable to that of another nonglass-forming liquid, $l-\mathrm{ZrO}_{2}$, and to that of crystalline $\mathrm{Er}_{2} \mathrm{O}_{3}$. The formation of distorted $\mathrm{OEr}_{4}$ tetraclusters in the liquid is confirmed, while $\mathrm{OZr}_{3}$ triclusters are dominant in $l-\mathrm{ZrO}_{2}$ and $\mathrm{OAl}_{3}$ triclusters dominate in another $\mathrm{A}_{2} \mathrm{X}_{3}$ non-GFL, $l-\mathrm{Al}_{2} \mathrm{O}_{3}$. Apparently, the formation of a distorted tetracluster network in $l-\mathrm{Er}_{2} \mathrm{O}_{3}$ gives rise to a long periodicity, yielding the sharp principal peak. This long-range periodicity originates from the significantly increased weight at $\sim 180^{\circ}$ in the Er-O-Er bond angle distribution, suggesting that the arrangement of distorted $\mathrm{OEr}_{4}$ tetraclusters involves nearly linear connections, which are not observed in other oxide liquids. Furthermore, persistent homology suggests that $l-\mathrm{Er}_{2} \mathrm{O}_{3}$ is homologically similar to the crystalline phase and that both phases are very densely packed, in contrast to a typical GFL such as $l-\mathrm{SiO}_{2}$. This similarity is presumed to be the signature of the liquid, and a considerable difference between two $\mathrm{A}_{2} \mathrm{X}_{3}$-type oxide liquids, $l-\mathrm{Al}_{2} \mathrm{O}_{3}$ and $l$ $\mathrm{Er}_{2} \mathrm{O}_{3}$, is uncovered. The additional DF-MD simulations demonstrate that $l-\mathrm{Er}_{2} \mathrm{O}_{3}$ has an electronic band gap of $0.6 \mathrm{eV}$, which is considerably lower than that of $c-\mathrm{Er}_{2} \mathrm{O}_{3}$ due to angular distortions and nearly linear connections within the polyhedral network. The dynamics of atoms show pronounced mobility, as evidenced by the MSDs, resulting in a very low viscosity, and thus place $l-\mathrm{Er}_{2} \mathrm{O}_{3}$ within the regime of extremely fragile liquids.

\section{Acknowledgements}

The synchrotron radiation experiments were performed at BL04B2 of SPring-8 with the approval of the Japan Synchrotron Radiation Research Institute (JASRI) (Proposal No. 2016A0134). This research was supported by JST PRESTO, Japan Grant Numbers JPMPR15N4 (to S.K.); the "Materials Research by Information Integration" initiative $\left(\mathrm{MI}^{2} \mathrm{I}\right)$ project of the Support Program for Starting Up Innovation Hub from JST (to Y.O., S.T., S.K., At.M., and Y.H.); JST CREST 15656429 (to Y.H.); JSPS KAKENHI Grant Number JP17H03121 (to A.M.); and the TIA collaborative research program "Kakehashi", TK19-004 (to S.K.). D.R.S. and S.M.S. acknowledge the Research Council of Norway (FRINATEK Project No. 275139/ F20) for their financial support and UNINETT Sigma2 (Project No. NN9264K) for providing computational resources. The density measurement experiments were supported by the ISS crew members and ground operation staff.

\section{Author details}

${ }^{1}$ Human Spaceflight Technology Directorate, Japan Aerospace Exploration Agency (JAXA), Tsukuba 305-8505, Japan. ${ }^{2}$ Department of Physics and Earth Sciences, Faculty of Science, University of the Ryukyus, Nakagami-gun, Okinawa 903-0213, Japan. ${ }^{3}$ Center for Materials Research by Information Integration $\left(\mathrm{CMI}^{2}\right)$, Research and Services Division of Materials Data and Integrated System (MaDIS), National Institute for Materials Science (NIMS), Ibaraki 305-0047, Japan. ${ }^{4}$ Research Center for Advanced Measurement and Characterization, NIMS, Sayo-gun, Hyogo 679-5148, Japan. ${ }^{5}$ PRESTO, Japan Science and Technology Agency, Chiyoda-ku, Tokyo 102-0076, Japan. ${ }^{6}$ Diffraction and Scattering Division, Japan Synchrotron Radiation Research Institute (JASRI), Sayo-gun, Hyogo 679-5198, Japan. Institute for Integrated Radiation and Nuclear Science, Kyoto University, Sennan-gun, Osaka 590-0494, Japan. ${ }^{8}$ Department of Materials Science and Engineering, NTNU Norwegian University of Science and Technology, Trondheim 7491, Norway. ${ }^{9}$ Department of Physics, NTNU Norwegian University of Science and Technology, Trondheim 7491, Norway. ${ }^{10}$ Computational Physics Laboratory, Tampere University, P.O. Box 692, Tampere 33014, Finland. ${ }^{11}$ Institute of Space and Astronautical Science, JAXA, Tsukuba 305-8505, Japan. ${ }^{12}$ SOKEN-DAI (The Graduate University for Advanced Studies), Sagamihara 252-5210, Japan. ${ }^{13}$ Graduate School of Science and Technology, Hirosaki University, Bunkyo-cho, Hirosaki 036-8561, Japan. ${ }^{14}$ National Institute of Technology, Hakodate College, Hakodate, Hokkaido 042-0953, Japan. ${ }^{15}$ Institute for Materials Research, Tohoku University, Aoba-ku, Sendai 980-8577, Japan. ${ }^{16}$ Advanced Engineering Services Co., Ltd., Tsukuba, Ibaraki 305-0032, Japan. ${ }^{17}$ Center for Advanced Intelligence Project, RIKEN, Nihonbashi, Chuo-ku, Tokyo 103-0027, Japan. ${ }^{18}$ WPI Advanced Institute for Materials Research, Tohoku University, Sendai 980-8577, Japan.

${ }^{19}$ Kyoto University Institute for Advanced Study, WPI-ASHBi, Kyoto University, Kyoto 606-8501, Japan. ${ }^{20}$ CREST, Japan Science and Technology Agency,

Gobancho, Chiyoda-ku, Tokyo 102-0076, Japan

\section{Author contributions}

S.K. and C.K. designed this research. The high-energy $\mathrm{X}$-ray diffraction measurements were performed by S.K., C.K, Y.O., At.M., AK.M., Y.W., K.O., H.T., and J.T.O. The density measurements were conducted by C.K., T.I, Y.W., Y.N., H. T., and H.O. The classic molecular dynamics simulations were performed by S.T. The density functional - molecular dynamics simulations were conducted by D.R.S., S.M.S., and J.A.; S.K., D.R.S., S.M.S., J.A., I.O., Y.H., and O.S. analyzed the data, and S.K., C.K., and J.A. wrote the article.

\section{Data availability}

The datasets generated during and/or analyzed during the current study are available from the corresponding author upon reasonable request.

\section{Conflict of interest}

The authors declare that they have no conflict of interest.

\section{Publisher's note}

Springer Nature remains neutral with regard to jurisdictional claims in published maps and institutional affiliations.

Supplementary information is available for this paper at https://doi.org/ 10.1038/s41427-020-0220-0.

Received: 23 August 2019 Revised: 26 March 2020 Accepted: 1 April 2020. Published online: 2 June 2020

\section{References}

1. Zachariasen, W. H. The atomic arrangement in glass. J. Am. Chem. Soc. 54, 3841-3851 (1932).

2. Sun, K-H. Fundamental condition of glass formation. J. Am. Ceram. Soc. 30, 277-281 (1947). 
3. Angell, C. A. Formation of glasses from liquids and biopolymers. Science $\mathbf{2 6 7}$, 1924-1935 (1995).

4. Greaves, G. N. \& Sen, S. Inorganic glasses, glass-forming liquids and amorphizing solids. Adv. Phys. 56, 1-116 (2007)

5. Salmon, P. S. \& Zeidler, A. Identifying and characterising the different structural length scales in liquids and glasses: an experimental approach. Phys. Chem Chem. Phys. 15, 15286-15308 (2013).

6. Price, D. L. High-Temperature Levitated Materials (Cambridge University Press, 2010).

7. Ansell, S. et al. Structure of liquid aluminum oxide. Phys. Rev. Lett. 78, 464-466 (1997).

8. Landron, C. et al. Liquid alumina: detailed atomic coordination determined from neutron diffraction data using empirical potential structure refinement. Phys. Rev. Lett. 86, 4839-4842 (2001).

9. Krishnan, S. et al. Structure of normal and supercooled liquid aluminum oxide. Chem. Mater. 17, 2662-2666 (2005).

10. Shi, C. et al. The structure of amorphous and deeply supercooled liquid alumina. Front. Mater. 6, 38 (2019).

11. Skinner, L. B. et al. Joint diffraction and modeling approach to the structure of liquid alumina. Phys. Rev. B 87, 024201 (2013).

12. Jahn, S. \& Madden, P. A. Structure and dynamics in liquid alumina: simulations with an ab initio interaction potential. J. Non-Cryst. Solids 353, 3500-3504 (2007)

13. Vashishta, P., Kalia, R. K. Nakano, A. \& Rino, J. P. Interaction potentials for alumina and molecular dynamics simulations of amorphous and liquid alumina. J. Appl. Phys. 103, 083504 (2008).

14. Skinner, L. B. et al. Molten uranium dioxide structure and dynamics. Science 346, 984-987 (2014).

15. Alderman, O. L. G. et al. Corium lavas: structure and properties of molten $\cup_{2^{-}}$ $\mathrm{ZrO}_{2}$ under meltdown conditions. Sci. Rep. 8, 2434 (2018).

16. Hong, Q. J. et al. Combined computational and experimental investigation of high temperature thermodynamics and structure of cubic $\mathrm{ZrO}_{2}$ and $\mathrm{HfO}_{2}$. Sci. Rep. 8, 14962 (2018).

17. Skinner, L. B. et al. Low cation coordination in oxide melts. Phys. Rev. Lett. 112 157801 (2014)

18. Kohara, S. et al. Atomic and electronic structures of an extremely fragile liquid. Nat. Commun. 5, 5892 (2014).

19. Tamaru, H. et al. Status of the electrostatic levitation furnace (ELF) in the ISSKIBO. Microgravity Sci. Technol. 30, 643-651 (2018).

20. Kohara, S. et al. Synchrotron X-ray scattering measurements of disordered materials. Z. Phys. Chem. 230, 339-368 (2016).

21. Kohara, S., Ohara, K., Ishikawa, T., Tamaru, H. \& Weber, R. Investigation of structure and dynamics in disordered materials using containerless techniques with in-situ quantum beam and thermophysical property measurements. Quantum Beam Sci. 2, 5 (2018).

22. Kohara, $\mathrm{S}$. et al. Structural studies of disordered materials using high-energy $x$ ray diffraction from ambient to extreme conditions. J. Phys. Condens. Matter. 19, 506101 (2007)

23. Faber, T. E. \& Ziman, J. M. A theory of the electrical properties of liquid metals. Philos. Mag. 11, 153-173 (1965).

24. Greben, O., Jóvári, P., Temleitner, L. \& Pusztai, L. A new version of the RMC++ Reverse monte carlo programme, aimed at investigating the structure of covalent glasses. J. Optoelectron. Adv. Mater. 9, 3021-3027 (2007).
25. Blöchl, P. E. Projector augmented-wave method. Phys. Rev. B 50, 17953-17979 (1994).

26. Kresse, G. \& Joubert, D. From ultrasoft pseudopotentials to the projector augmented-wave method. Phys. Rev. B 59, 1758-1775 (1999).

27. Kresse, G. \& Furthmüller, J. Efficient iterative schemes for ab initio total-energy calculations using a plane-wave basis set. Phys. Rev. B 54, 11169-11186 (1996).

28. Perdew, J. P., Burke, K. \& Ernzerhof, M. Generalized gradient approximation made simple. Phys. Rev. Lett. 77, 3865-3868 (1996).

29. Krukau, A. V., Vydrov, O. A., Izmaylov, A. F. \& Scuseria, G. E. Influence of the exchange screening parameter on the performance of screened hybrid functionals. J. Chem. Phys. 125, 224106 (2006).

30. Henkelman, G., Arnaldsson, A. \& Jónsson, H. A fast and robust algorithm for Bader decomposition of charge density. Comput. Mater. Sci. 36, 354-360 (2006).

31. Sanville, E., Kenny, S. D., Smith, R. \& Henkelman, G. Improved grid-based algorithm for Bader charge allocation. J. Comput. Chem. 28, 899-908 (2007).

32. Tang, W., Sanville, E. \& Henkelman, G. A grid-based Bader analysis algorithm without lattice bias. J. Phys. Condens. Matter 21, 084204 (2009).

33. Nosé, S. A unified formulation of the constant temperature molecular dynamics methods. J. Chem. Phys. 81, 511-519 (1984).

34. Hiraoka, Y. et al. Hierarchical structures of amorphous solids characterized by persistent homology. Proc. Natl Acad. Sci. USA 113, 7035-7040 (2016).

35. https://www.wpi-aimr.tohoku.ac.jp/hiraoka_labo/homcloud/index.en.htm

36. Aksay, I. A., Pask, J. A. \& Davis, R. F. Densities of $\mathrm{SiO}_{2}-\mathrm{Al}_{2} \mathrm{O}_{3}$ melts. J. Am. Ceram. Soc. 62, 332-336 (1979)

37. Paradis, P. F., Ishikawa, T., Saita, Y. \& Yoda, S. Non-contact thermophysical property measurements of liquid and undercooled alumina. Jpn J. Appl. Phys. 43, 1496-1500 (2004).

38. Mei, Q., Benmore, C. J. \& Weber, J. K. R. Structure of liquid $\mathrm{SiO}_{2}$ : a measurement by high-energy x-ray diffraction. Phys. Rev. Lett. 98, 057802 (2007).

39. Salmon, P. S., Martin, R. A., Mason, P. E. \& Cuello, G. J. Topological versus chemical ordering in network glasses at intermediate and extended length scales. Nature 435, 75-78 (2005).

40. Salmon, P. S. Magma under Pressure: Advances in High-Pressure Experiments on Structure and Properties of Melts (Ed. Kono, Y. \& Sanloup, C.) pp. 347 (Elsevier, Amsterdam, 2018)

41. Shannon, R. D. \& Prewitt, C. T. Effective ionic radii in oxides and fluorides. Acta Cryst. B25, 925-946 (1969).

42. Onodera, Y. et al. Understanding diffraction patterns of glassy, liquid and amorphous materials via persistent homology analyses. J. Ceram. Soc. Jpn 127, 853-863 (2019).

43. Saiki, A., Ishizawa, N., Mizutani, N. \& Kato, M. Structural change of C-rare earth sesquioxides $\mathrm{Yb}_{2} \mathrm{O}_{3}$ and $\mathrm{Er}_{2} \mathrm{O}_{3}$ as a function of temperature. Yogyo Kyokai Shi 93, 649-654 (1985).

44. Bhatia, A. B. \& Thornton, D. E. Structural aspects of the electrical resistivity of binary alloys. Phys. Rev. B 4, 3004-3012 (1971).

45. Kohara, S. et al. Relationship between topological order and glass forming ability in densely packed enstatite and forsterite composition glasses. Proc. Natl Acad. Sci. USA 108, 14780-14785 (2011).

46. Akola, J. et al. Network topology for the formation of solvated electrons in binary $\mathrm{CaO}-\mathrm{Al}_{2} \mathrm{O}_{3}$ composition glasses. Proc. Natl Acad. Sci. USA 110 10129-10134 (2013). 